\title{
Enterprise Architecture Cybernetics and the Edge of Chaos: Sustaining Enterprises as Complex Systems in Complex Business Environments
}

\author{
Hadi Kandjani \\ Centre for Enterprise \\ Architecture Research and \\ Management (CEARM), School \\ of ICT, Griffith University, \\ Brisbane, Australia \\ H.Kandjani@grifith.edu.au
}

\author{
Peter Bernus \\ Centre for Enterprise \\ Architecture Research and \\ Management (CEARM), School \\ of ICT, Griffith University, \\ Brisbane, Australia \\ P.Bernus@griffith.edu.au
}

\author{
Sue Nielsen \\ Institute for Integrated and \\ Intelligent Systems (IIIS), \\ School of ICT, \\ Griffith University, \\ Brisbane, Australia \\ S.Nielsen@griffith.edu.au
}

\begin{abstract}
The purpose of this paper is primarily theoretical to propose and detail a model of system evolution, and show its derivation from the fields of Enterprise Architecture, cybernetics and systems theory. Cybernetic thinking is used to develop a 'Co-evolution Path Model' to explain how enterprises co-evolve with their environments. The model is re-interpreting Stafford Beer's Viable System Model, and also uses Conant and Ashby's theorem of the 'good regulator', exemplifying how various complexity management theories could be synthesised into a cybernetic theory of Enterprise Architecture - informing management of mechanisms to maintain harmony between the evolution of the enterprise as a system and the evolution of its environment.
\end{abstract}

\section{Introduction}

The increasing complexity of the IT and business environment, and the need to ensure alignment of IT with business goals and operations, have given rise to a number of initiatives in information systems research and practice [1]. Prominent amongst these is the discipline of Enterprise Architecture which is now widely accepted as a requirement for high level and comprehensive management of the IT enterprise [2].

Despite this acceptance, the field of Enterprise Architecture is still undergoing investigation into its theoretical basis, with considerable work focused on elaborating and harmonising the various frameworks and models. This paper aims to contribute to this work by exploring the application of cybernetic thinking to explain how systems co-evolve with their environments. A 'Co-evolution Path Model' is developed which reinterprets 'System 5' of Beer's Viable System Model [3], i.e., the system which is responsible for strategically steering the organisation.
The paper uses the GERAM framework [4, 34, 35] as a basis for the model, because of it 'agnostic' nature and its important concepts of Life History and Life Cycle. GERAM defines a comprehensive set of concepts to represent and explore enterprise evolution. GERAM is a "toolkit of concepts for designing and maintaining enterprises for their entire life history" (ibid) and the objective of this framework is "to systematise various contributions of the field that address the creation and sustenance through life of the enterprise as a complex system”. GERAM is different from other frameworks (those developed for only pragmatic purposes), as pragmatic frameworks do not necessarily have to make certain theoretically important differentiations, nor have to be complete from every respect to be usable for some particular practical EA project. However, fundamental conceptual differentiations and domain completeness are needed when it comes to the use of an EA framework to interpret models of evolution and management in general, so as to create a foundation of what we call 'EA Cybernetics'. While many frameworks could be made complete by satisfying all ISO15704 requirements [36, 37], we use GERAM's concepts as they already have the desired properties.

The purpose of this paper is primarily theoretical to propose and detail a model and show its derivation from the fields of Enterprise Architecture, Cybernetics and Systems Theory. Since the development of this model is in its early stages, it has yet to be tested in empirical studies. However, it proposes both "testable propositions and causal explanations" [5] which may be applied to real cases.

In practical terms, such a model might enable organisations to recognise the signs of dissonance between system complexity and environment complexity and as a result make deliberate decisions to steer away both from system states that are the edge of (perceived) chaos and trends to obsolescence. The 
paper also proposes the development of an EA cybernetics framework which can equally represent evolutionary and deliberate/designed changes.

Note that there are two forces at work: a system needs to be able to display complex enough behaviour to respond to the needs of (or survive in) the environment; at the same time excessive complexity makes the system hard to control. Part of the question is how complexity can be measured and how excessive complexity can be eliminated. One important complexity measure is the system's information content, as defined by the system's Kolmogorov complexity (KC) [44]. For example, the authors previously demonstrated [45], how known approximation methods of $\mathrm{KC}$ can be used to estimate the information content of a system and how this can be utilised to make architectural design decisions to 'design out' excessive complexity from a supply chain.

\section{Complexity and the Cybernetics Perspective}

Enterprises are best understood as intrinsically complex adaptive living systems: they can not purely be considered as 'designed systems', because deliberate design/control episodes and processes ('enterprise engineering', using models) are intermixed with emergent change episodes and processes (that may perhaps be explained by models). The mix of deliberate and emerging processes can create a situation in which the enterprise as a system is in dynamic equilibrium (for some stretch of time) - a property studied in General Systems Theory [6].

The evolution of the enterprise (or enterprises, networks, industries, the entire economy, society, etc) includes the emergent as well as the deliberate aspects of system change, therefore we believe that EA needs to interpret previous research in both. This is summarised as the main aim of the enterprise architecture discipline and practice, i.e., to explain change in enterprises as complex systems (through theory, models and methodologies) [7].

In response to the problem of managing complexity and fast change, many studies applied the cybernetic perspective to Enterprise Architecture (application of cybernetic concepts to EA management [8] and to EA principles e.g. as embodied in TOGAF [9]).

Stafford Beer believed that the dynamics of enterprises is about "the manipulation of men, material, machinery and money: the four M's”, but also about a fundamental manipulation of systems, which we call the "management of complexity" [10,3].

Norbert Wiener defined cybernetics as "the science of control and communication in the animal and machine" [11]. Ashby also calls it as the art of "steermanship", the study of coordination, regulation and control of systems, and argued that "truths of cybernetics are not conditional on their being derived from some other branch of science" [12].

Therefore the field embraces a set of self-contained groundings and foundations [12]. Ashby addressed the complexity of a system as one of the peculiarities of cybernetics and indicated that cybernetics must prescribe a scientific method of dealing with complexity as a critical attribute of a system.

Beer was the first person applying cybernetics to management and defined cybernetics as "the science of effective organisation" $[13,14]$. He was also first to coin the word "Management Cybernetics" - a field applying cybernetic laws and theories to all types of socio-technical organisations / “enterprises” [15].

Beer elaborates on the relevance of cybernetics to management in 'Cybernetics and Management' [13] and describes his first discoveries and promises in the management discipline. He also characterises cybernetics as "the science of control" and management as "the profession of control" [10].

Therefore EA research has acknowledged the relevance of cybernetics for modern enterprises which cannot expect to build 'ideal' and one-time systems but must undertake continuous steering and control of their evolving systems [16]. Such a perspective elaborates on Beer's 'system 4 and 5' to cope with the increasing complexity of organisations and their environments.

\section{Enterprise Architecture Cybernetics}

One common topic of cybernetics is the treatment of complexity (whether it is the complexity of the structure, behaviour, control, management, or other relevant view of the system of interest), raising the question how systems can be managed, controlled, changed, designed, or partially influenced for producing emergent adaptive behaviour.

A distinct problem, characteristic of complex systems, is (by definition of what constitutes a complex system) that none of these tasks can be based on a complete predictive model, therefore the involved decisions must be based on incomplete information. Due to this character of complex systems we need theories and methods, or structures, that produce such self-control behaviour (either in deliberate or in emergent way). Whichever way this control is exercised, it should be able to be described by an external observer as 'partial control' that nevertheless achieves a set of valued system properties (such as sustainability, viability, availability, and so on).

For the above reason, any controller (on any level of a system that is characterised as complex on that 
level) only has, or can only have, an incomplete model of the system, and sees the system through this model to make decisions to control that system. The complexity of a model like this is the 'apparent complexity' of the system from the given controller's (manager's) point of view.

Checkland warns that theories, frameworks and models with an excessive level of abstraction and general systems principles of 'wholeness' could be in danger of not being able to deal with real practice [17]. At the same time there exist very specific and contextdependent theories, frameworks and models which sacrifice generality and abstraction, on the other hand often there is little guidance on the limits of their applicability. The optimum degree of generality is somewhere in-between with different levels of abstraction for each purpose. For example, the aim of General Systems Theory [6] is not achievable by a single science discipline in isolation [18].

In order to develop a model which may explain how systems co-evolve with their environments, we have adopted fundamental concepts of the Generalised Enterprise Reference Architecture and Methodology (GERAM) framework [4].

EA frameworks such as GERAM acknowledge that the optimum degree of generality is problem domain dependent, therefore it is necessary to provide a modelling framework that represents this continuum from the most generic to the most specific.

GERAM defines a) Generic Enterprise Modelling Concepts (GEMCs) [practically ontological theories], b) Partial Enterprise Models (PEMs) [usually in form of reference models] and c) Particular Enterprise Models (EMs). GEMCs define and formalise the most generic concepts of enterprise modelling, PEMs capture characteristics common to categories of enterprise entities within or across one or more industry sectors, and particular enterprise Models (EMs) that represent a particular enterprise entity [4]. EA Cybernetics must maintain an 'optimum degree of generality' to provide the discipline and practice with the 'right level of abstraction' for each purpose, whereupon given the abstract theory and a concrete system (and concrete problem), there should exist methods that can be used to solve or explain the problem, and achieve this within the limitations of available resource- and time constraints.

Enterprise Architecture, as a developing discipline, needs a model for theory development, testing and knowledge creation. Anderton and Checkland [19] developed a model of any developing discipline to demonstrate the cyclic interaction between theory development and formulation for a problem and theory testing $[19,20]$. For EA to be a developing discipline (Fig.1), we consider real world enterprise problem domains as the source of a development process, a source of issues to be addressed by theories, models and methods in enterprise related disciplines.

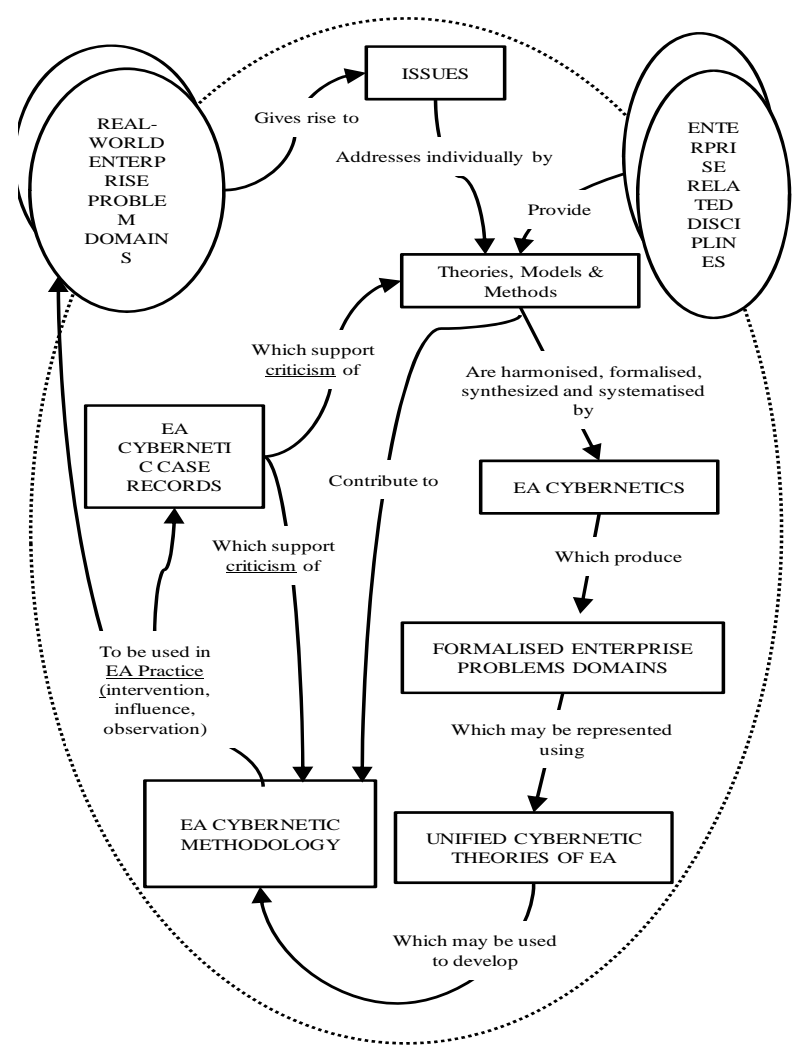

Figure 1. Enterprise Architecture as a Developing Discipline based on the model of activities and results of developing disciplines $[19,20]$

These will shape ideas by which two types of theories could be developed [20]:

a) substantive theories derived from related disciplines to apply relevant models, theories and methods in the problem domains, and

b) methodological theories about how to individually apply enterprise related disciplines in the problem domains.

Once we developed such theories, it is possible to state problems - not only existing problems in concrete enterprise problem domains, but also formalised, harmonised and synthesised problem statements by EA cybernetics within a new theory.

As a new theory, EA cybernetics produces formalised enterprise problem domains which may be represented using the unified cybernetic theory of EA. These unified theories may be used to develop a methodology(ies) to be used in EA practice.

Results of such synthesis must be tested in practice (through intervention, influence, or observation) to create 'case records', which in turn provide the source 
of criticism allowing better theories to be formulated (and better models, techniques, and methodologies). The application of the latter methodologies should be documented in case records which provide feedback to improve the individual- and the unified theories.

The EA discipline not only embraces the models, methods and theories of management and control - it also uses the same of systems engineering, linguistics, cognitive science, environmental science, biology, social science and artificial intelligence.

What cybernetic thinking is able to do is to provide a method of unifying (and relating) the contribution of these disciplines: cybernetic thinking can be used to represent the essence of multiple theories using abstract functions and processes (and meta-processes) and their relationships, rules and axioms (likely to be expressed in suitably selected logics).

Fig.1., shows the pathway through which the apport of these disciplines is formalised, synthesised, harmonised, systematised and eventually represented as a unified Cybernetic theory of EA. The Coevolution Path Model introduced in the next sections is an example of a cybernetic model of the control and management of viable complex systems that operate in complex environments.

\section{The Co-evolution Path Model: Dynamic Homeostasis vs. Dynamic Hetereostasis: An Example of an EA Cybernetics Model}

A key property of a viable system and a "measure of its submission to the control mechanism" is its ability to maintain homoeostasis, defined by Beer [10] as "constancy of some critical variable (output)".

In our model of co-evolution, we demonstrate the dynamic sustenance of requisite variety based on Ashby's law: "only variety can destroy variety" [12], paraphrased by Beer [21] as "variety absorbs variety", where 'variety' is the number of possible states of a system [22], or as recently clarified as the number of relevant states of a system [23].

In order for a system to dynamically achieve and maintain requisite variety and to be in dynamic equilibrium, the system requires communication channels and feedback loops. These communication channels serve as self-perpetuating mechanism and include both attenuation and amplification mechanisms. (Note that for the discussion below what we call a 'system' includes the system's controller.)

Considering the system and its environment as two coupled entities, if one component is perturbed, the effect of that perturbation on the other component is either amplified through positive feedback, or may be reversed (attenuated) through negative feedback.

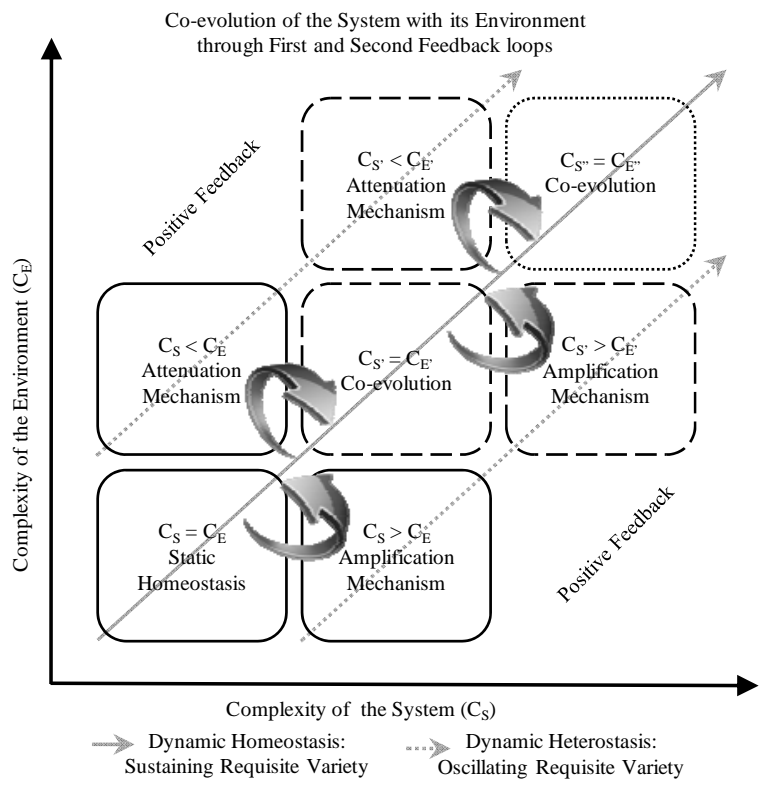

Figure 2. The Co-evolution Path Model

The role of a negative feedback loop is to reverse the effect of the initial perturbation and restore system homeostasis (in which critical variables are stable) while positive feedback can create unstable states [24].

We observe that both a system and its environment (including systems in that environment) evolve, and this can create imbalance between the requisite variety (maintained by the controller) of our system of interest and the variety that would be required for it to maintain homeostasis. In other words, systems that want to live long must co-evolve with their environment.

Formally: we consider the environment an entity with a possible set of observable states and if two such states require different response from the system then the system must be able to differentiate between them (thus they are two different relevant states). (Note: we may not be able to describe the environment as a system, although it may contain one or more systems.)

Consequently, in Fig. 2, the complexity of a system (CS) is defined to be the complexity of the model the controller of the system maintains (appears to be maintaining) so as to manage the system's operations Including the need to interact with the environment.

The complexity of the system's environment (CE) is a relative notion and is defined to be the complexity of the model of the environment that the controller of the system would need in order to maintain the system's homeostasis; - although it is sufficient if in the eyes of an external observer the system's controller appears to be maintaining such model.

Specifically, an 'environment model' must have predictive capability, so that the system, while interacting with its environment, can maintain a 
homeostatic trajectory. An environment model would thus include as components a) models of external systems with which our system interacts (including models of their controllers and operations), and b) a model of the rest of the environment, so as to be able to represent and predict the states of signals and resources among the system, the external systems and the rest of the environment: based on the theorem of the 'Good Regulator' [25], a good controller of a system must have a model of that system with an equal complexity at its disposal as the system to be controlled has.

In Fig 2, notice that:

1) If the complexity of the system (CS) equals to that of its environment (CE), then the system has the requisite variety and is in static equilibrium. However, any change in the complexity of the environment should be sensed by the system's self-perpetuating mechanism to restore the system to its initial state or to create a new equilibrium state;

2) If the complexity of the environment is greater than that of the system, then the system should attenuate the effects of this complexity, i.e., change and co-evolve with its environment (in other words, this happens when the environment produces, or is recognised to have the potential to produce, states in which the system can not function as expected);

3 ) If the complexity of the system is greater than that of its environment, then the system can potentially create a set of different states and perform behaviours which are not differentiated by its environment. The system can identify this extra complexity as undesired, or use an amplification mechanism to create new differentiations in the environment (e.g. marketing of new goods / services).

If a new enterprise lacks co-evolution mechanisms then it may be viable in the short term, but doomed in the long term. Such failure of enterprises is attributable to the inflexibility of their business models, due to the lack of attenuation and amplification mechanisms to sustain dynamic stability. This is why, according to Badalotti [26], the new economy's most successful start-ups have changed their business models several times in the first few years of their existence.

A successful example is America-on-line (AOL) that initiated its business and grew as an Internet Service Provider, but re-identified itself as a content provider, re-designing its business model and market positioning [26].

The Co-evolution Path Model has a level of abstraction that makes it applicable to any change and co-evolution of a complex system in its environment. In this paper we use concepts of GERA and of its Modelling Framework as they provide us with a comprehensive coverage of viewpoints through which no change in the environment would be neglected.
Using GERA concepts and viewpoints, the controller (manager in charge of the system) could

a) model and steer, in light of change in the environment, by taking into account relevant viewpoints of the environment's model, and

b) design co-evolution mechanisms to change or manipulate the system's operations, using a relevant combination of models, viewpoints and life cycle processes, as well as design a change trajectory in the system's life history.

For example, one could use the Strategic Alignment Model (SAM) by Henderson and Venkatraman [27] and map it to the Co-evolution Path Model: i.e., use SAM as a change/co-evolution model of the enterprise and its environment, using IT- and Business related viewpoints of our adopted modelling framework.

\section{Co-evolution Mechanisms: Order, complexity and the Edge of Chaos}

In this section, we demonstrate the transitions caused by changes in the complexity of a system (CS) and the complexity of its environment (CE), and relevant mechanisms to keep the system in equilibrium.

\subsection{Co-evolving/viable System states}

Consider the system in state 1 as its initial state where (approximately) CS = CE, i.e., the system is in a homeostatic state. If there is an increase in CE from state 1 to 2 (such as introduction of a substitute product or service to the market by a rival company) then this makes the company move into the vulnerable zone.

In order for the system to adapt to the changes in the environment and achieve the requisite variety and to remain on the viable path, it actually always needs some excess (but not excessive) complexity.

There are two different complexities here: the variety of the system and how the system achieves that variety (for instance, by the reconfiguration of its structure, acquiring more resources, creating new capabilities, reducing production costs, or innovating a new product or service).

Such adjustment which takes into account new differentiations (and thereby variety) in the environment will move the company from state 2 to state 3 where CS equals CE and the company remains on the coevolving / viable system path.

The history of manufacturing has typical examples of the above effect. The initial manufacturing lines of mass production in the car industry were only able to produce a low variety of products, as the environment evolved, differentiated customer requirements made the market more sophisticated and companies with 
mass production systems were unable to respond to this new complexity. Approximately 15 20 years after world war II, manufacturing companies realised that greater flexibility is needed in terms of the variety of products that a manufacturing systems can produce.

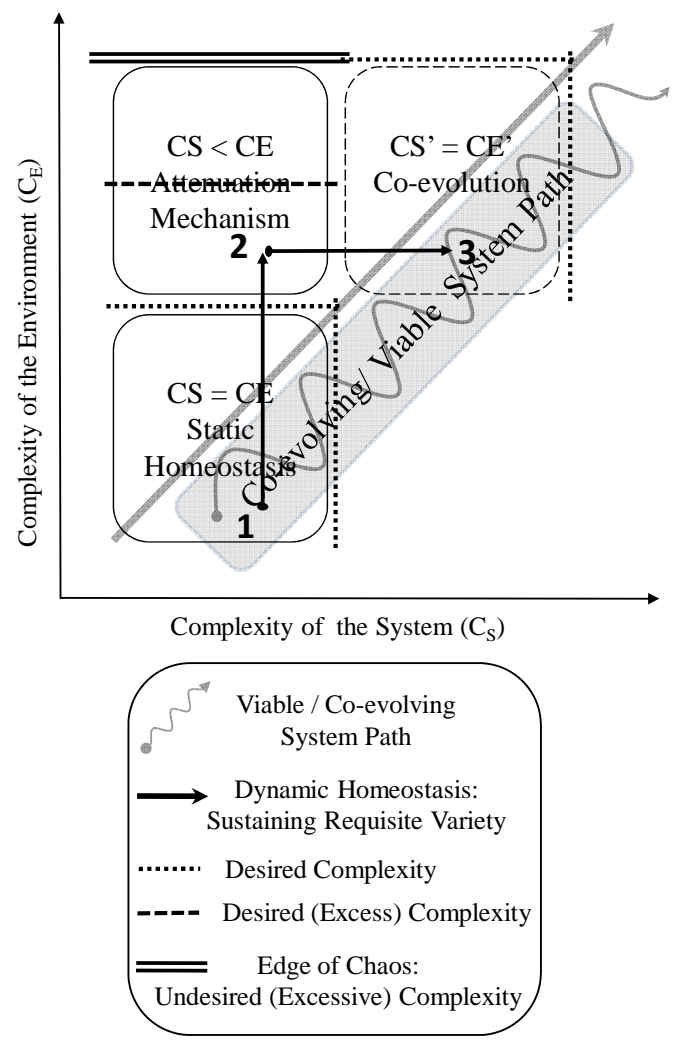

Figure 3. Traces (paths) of Co-evolving/viable System states

From the mid 1970s to the mid 1980s integrated manufacturing systems were built which had numerically controlled (NC) machines, such that the same machine would be able to perform many manufacturing operations (limited by the basic functions of the machines in the system). However, these integrated systems were not easy to change, i.e. while the complexity of responses these systems could give to market needs were far greater than that of transfer lines, incremental changes were not possible. Thus after a while these systems could not respond to changes in the environment's requests, and died out.

For the (manufacturing) system to be able to keep co-evolving with the environment, the next generation of flexible (cell based) manufacturing systems [41] had to be developed (using the group technology paradigm). These systems were able to be incrementally developed, old cells decommissioned and new cells added, as the needs of the environment dictated. The development gave rise to the manufacturing agility movement (1990s). This moved the centre of attention from the capability of the single company as a system to the network of enterprises that can create the requisite variety in a dynamic fashion, by forming so-called virtual enterprises on demand $[39,40]$. Another example from the continuous (small batch) chemical production industry is the 'pipeless' chemical factory invention [43]. The complex demands of the market are impossible to meet cost effectively by a conventional fixed-pipe factory, where the variety of ways the production equipment can be used to channel products is limited. The pipeless factory allows any equipment to be interconnected with any other equipment, using robot controlled flexible piping system, increasing the complexity of the behaviour this new type of chemical factory can display.

\subsection{Inefficient System states}

Now consider the state where the system has, or acquires, excessive resources and capabilities (a set of potential structures and ability that could perform functions that are not 'completely' invoked by the system's environment).

This transition from state 4 to state 5 creates undesired or unnecessary (excessive) complexity. For example, a manufacturing company (in state 4) with a leading R\&D department designs a new product, the inbound logistics provides necessary goods and materials and finally the company establishes its production line (state 5). However, if the market is not ready for such product differentiation, the company has excessive, and therefore undesired, complexity.

As the controller (management) has difficulty being able to predict what level of complexity will the market / environment embrace, there must exist mechanisms to recognise and curtail excessive systems complexity.

To reduce the potential risks and inefficiency of this new venture as a new potential structure and to increase the probability of success when designing change projects, this company should apply complexity reduction techniques such as Axiomatic Design $[28,29,30]$ to reconfigure its functions, structures and architectures to shift from the inefficiency zone and achieve effectiveness and efficiency in state 6 where company has some desired excess complexity but expels excessive complexity.

At this stage, the company still has excess complexity which should be amplified to the market ('the environment'), therefore the marketing department should apply effective marketing strategies in order to introduce the new product to the market and promote its sales.

Having developed and implemented a successful marketing strategy and plan, the manufacturing 
company amplifies its excess desired complexity caused by new structures and ends up in a new homeostatic state (state 7).

For example, many Japanese electronic goods manufacturers usually release a wide variety of products domestically, to test customer reaction, but based on the market response reduce the variety of products before marketing world-wide and simplify the supply chain in case of global production. Such companies compete on variety being released to the market fast [42], while carefully managing how much variety they offer, when and where.

\subsection{Vulnerable System states}

Let us consider the state where there is an increase in the complexity of the environment (state 8 to 9).

For the system to respond to the changes in $\mathrm{CE}$ and achieve requisite variety (and a new homeostasis), and remain viable, it needs to attenuate more complexity and co-evolve with the environment.

For example, rivals can reduce the company's market share by introducing new or substitute products and therefore the company is at the risk of losing its competitive advantage. The company has no other choice than reconfigure its current resources and structures, or acquire more resources and potential structures to create new capabilities / competencies and re-vitalis its competitive advantage (perhaps merging with other companies to survive: state $9 \rightarrow 10$ ).

Unfortunately, state transitions from state 9 to 10 usually impose excessive complexity on the company. E.g. companies may underestimate the integration tasks in horizontal mergers and can end up with an inefficient operation preventing them from enjoying the benefits of the merger [38]. Therefore to avoid inefficiency and mitigate risks of establishing a new production line (new structures and capabilities, createing undesired excessive complexity) the R\&D department / the design authority should apply complexity reduction methods and techniques such as 'Extended' Axiomatic Design Theory (EAD) [23].

EAD deals with reducing and possibly avoiding the complexity of the change process, which designs and implements necessary changes (such as the establishment of a new production line). Using this method, the company may avoid the inefficiency zone and remain in the viable zone.

The spiral arrow in Fig.3 demonstrates the viable system path in which the system dynamically sustains its homeostasis and avoids or rectifies inefficient and vulnerable states by invoking relevant attenuation and amplification mechanisms.

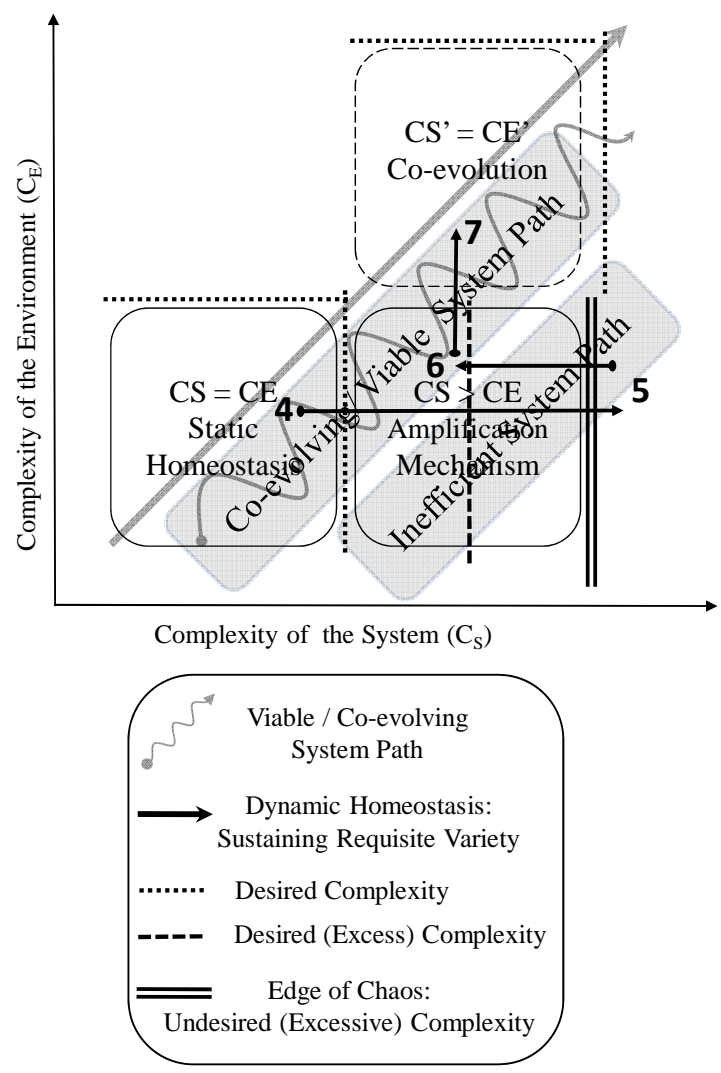

Figure 4. Traces of Inefficient System states

\subsection{Non-viable System states}

Let us now consider the states where the company will not remain viable (as in states 11 to state 12). Enterprises as live systems have a number of variables characterising essential survival properties. Ashby [31] refers to these as 'essential variables' (crucial to a system's survival) - modern literature would refer to these as strategic 'critical success factors'

Ashby defines survival as: “... a line of behaviour [that] takes no essential variable outside given limits" (Ashby 1960; Geoghegan and Pangaro 2009) [31,32]. Therefore, by definition, any line of behaviour outside limits of essential variables is on the non-viable system path and is fatal to the system's lifeline.

For a system to be regarded as adaptive, and therefore viable, Ashby introduces two necessary feedback loops (Ashby 1960; Geoghegan and Pangaro 2009; Umpleby 2009) [31,32, 33]. The first frequently operating loop makes small modifications and corrections to the system. The second loop in fact changes the structure and architecture of the system when the tolerance of the essential variables (invoked by dramatic changes in the system's environment) falls (or is predicted to be falling) beyond the limits of 
survival. If the system's second loop does not respond to the changes in complexity of the environment, then the system will be on the non-viable path.

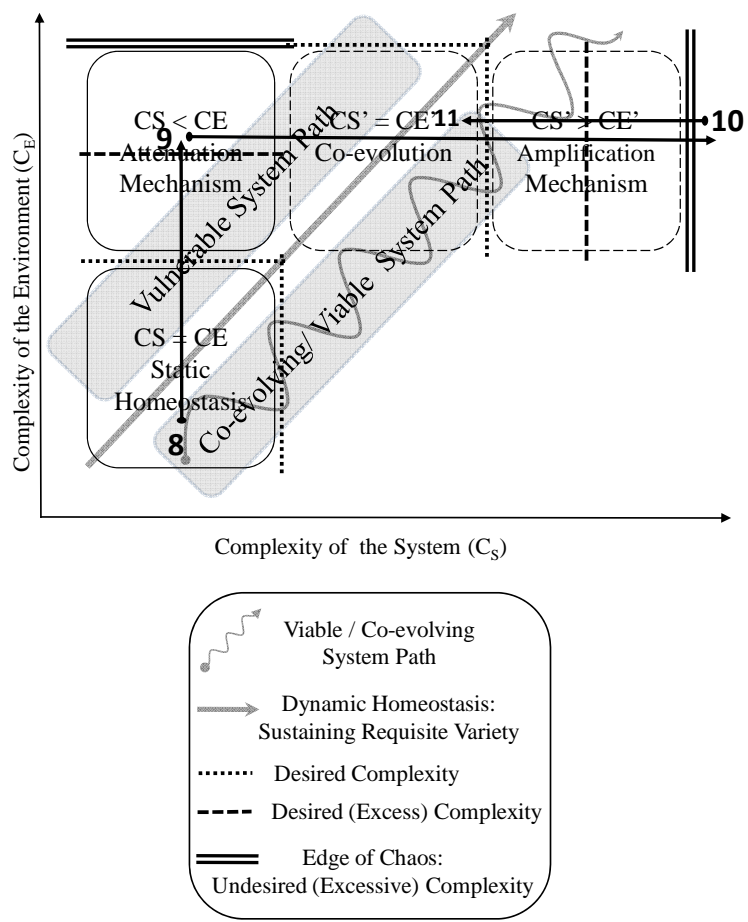

Figure 5. Traces of Vulnerable System states

Based on Ashby's theory of adaptation [31], Umpleby [33] indicates that the first feedback loop is necessary for a system to learn a pattern of behaviour which is necessary for a specific environment, while the second feedback loop is required for a system to identify the changes in the environment and design and create new patterns of behaviour.

If there is a dramatic increase in complexity of the environment (as in states 11 to state 12) it is possible that the system is not prepared to react due to scarcity of resources, lack of dynamic capability, inability to create new structures in a timely manner and adapt its architecture to the change in the environment.

The lack of an appropriate second feedback loop makes the system nonviable and the system is doomed to fail. If in this state, the company may save itself by establishing a partnership or merge (a reactive move, where the system relies on another system for rescue).

In summary, we can classify system states into four groups in terms of co-evolvability/ viability:

- Non-viable System states (States: 12-13-14)

- Vulnerable System states (States: 8-9-10-11)

- Inefficient System states (States: 4-5-6-7)

- Co-evolving/viable System states (States: 1-2-3)

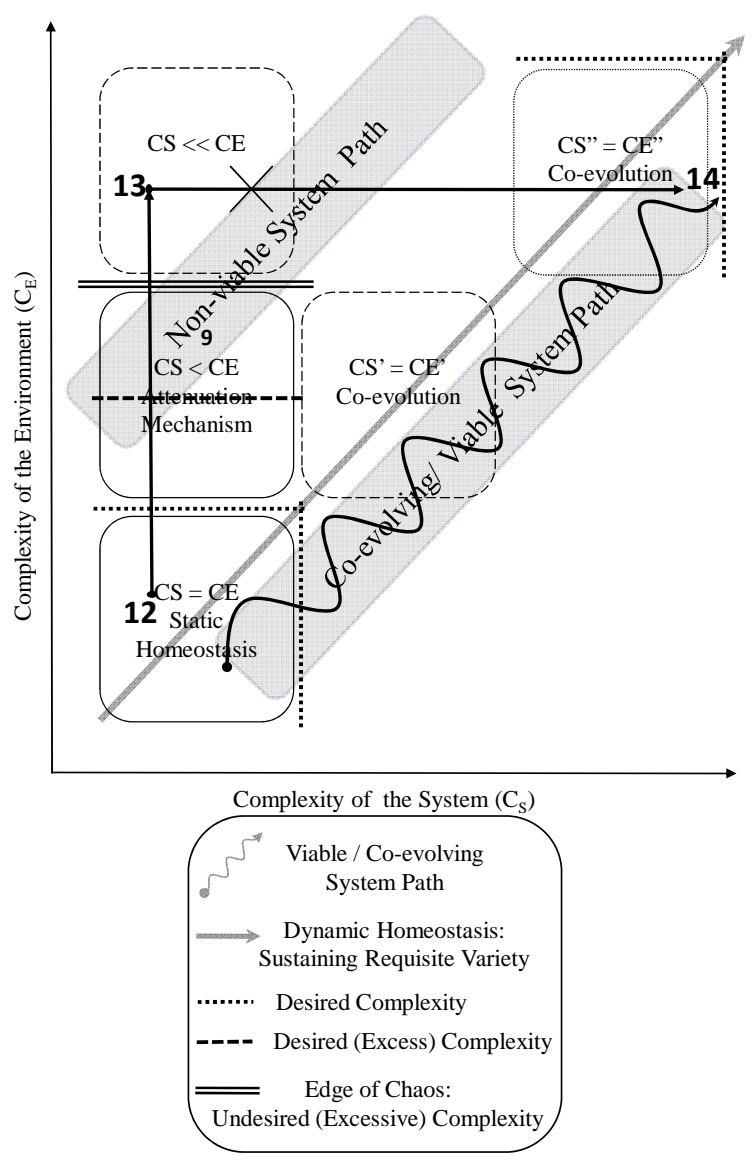

Figure 6. Traces of Non-viable System States

\subsection{Viability and EA Cybernetics}

The theory of viable systems argues for the need of a controller in a system ('system 5') to monitor the environment, so as to predict the need for change and steer away the system from a non viable trajectory. However, apart from the structural imperative, there is no theory or method proposed for this controller to use.

Ashby's law gives an argument for this system's existence (as the system is meant to be able to have enough variety so as to remain viable), however, no method or theory is given to achieve this or to measure variety. The two theories are even seemingly in contradiction, because while Ashby requires the system to have more variety than the environment, Beer points out that the system can never have a complete model of the environment. The co-evolution path model removes this contradiction, as it considers the system dynamics of evolution.

The first consequence is that the system (and its controller) only need to have a view of the environment and differentiate relevant states [23], thus the complexity of the model that the system needs to have of its environment is not the same as the complexity of 
the model of the environment that an omniscient observer would see. This allows the system to prepare for future unknown and unpredictable states of its surroundings, because the controller only needs partial proof about the future states of the environment.

For example, the manufacturing company is unable to predict exactly what products will be needed in the medium term, but it still can predict that all future products would be based on a number of known technology elements (given what is feasible today in the research laboratories). Thus the great number of actual combinations of product features do not add to the complexity of states of the environment.

The second consequence of the co-evolution path model is that it makes the imperative of excess complexity reduction explicit. The recognition of the need, combined with methods of complexity estimation methods [45], and a complexity reduction methodology (using extended axiomatic design [7]) form a synthesis of several disciplines, each contributing to the maintenance of a system on a viable path.

\section{Conclusion}

We proposed a Co-evolution Path Model using cybernetic principles to explore and map how organisations might manage complexity in light of (anticipated) changes in the environment. In proposing this model, it is understood that it is not possible to create complete models of large scale complex systems (such as an enterprise) or of the environment, and use them to fully control the system. This would deny the very complexity which we are attempting to address.

It is clear that we must accept that we can only have incomplete models of the complex systems that we want to control. Secondly we accept that when living organisms (such as people) are part of a system, their actions are not completely dictated by the system they are part of, nor are they necessarily guided by logic. Power relations, survival, self-interest, groupinterest, value systems, culture, etc are all participating in determining how a system 'plays out', in other words, however logical the design of a system may be, relying on the logic of processes is insufficient.

We believe that future research which explores the human and organizational implications of the cybernetic perspective would be useful when studying enterprises as complex systems.

The discipline of Cybernetics developed fundamental theories of complexity, therefore the authors propose 'EA Cybernetics' as a sub-discipline of EA, aiming to synthesise and harmonise the many pertinent cybernetic models developed over the past fifty years and apply them in the field of EA.
EA cybernetics is the re-interpretation of old- and new theories to understand their individual contributions, and to point at the need for genuinely new results. Cybernetic thinking provides a method of unifying / relating the apport of multiple disciplines. We expect that a synthesis would yield a new, unified cybernetic model of EA, more powerful theories, reference models, and methodologies than we have today, both in the problem domain and meta level (discipline development). we developed as an example an EA cybernetic model called the 'Co-evolution Path Model' that re-interprets 'System 5' of Beer's Viable System Model (based on Ashby's requisite variety law and first and second feedback loops) to dynamically sustain system viability.

\section{References}

[1] Luftmann, J., and R. Kempaiah, "An Update on businessIT alignment: "A Line” has been drawn," MIS Quarterly Executive (6:3), 2007, pp.165-177.

[2] Winter, R. and E.J. Sinz, "Enterprise Architecture (Editorial)," IS and e-Business Management (5:4), 2007, pp. 357-358

[3] Beer, S., Diagnosing the system for organizations, Wiley, 1985.

[4] IITF, GERAM: "Generalised enterprise reference architecture and methodology," v1.6.3. IFIP-IFAC Task-Force 1999, (also available as Chapter 2, Bernus, P., Nemes, L. and Schmidt,G. (eds.), Handbook on Enterprise Architecture, Berlin : Springer), 2003.

[5] Gregor, S., "The nature of theory in information systems," MIS Quarterly (30:3), 2006, pp. 611-642.

[6] Von Bertalanffy, L., General system theory: Foundations, development, applications, New York : Braziller, 1968.

[7] Kandjani, H. and P. Bernus, "Capability Maturity Model for Collaborative Networks based on Extended Axiomatic Design Theory," in Adaptation and Value Creating CNs, Camarinha-Matos, L., Pereira-Klen, A. and Afsarmanesh, $\mathrm{H}$. (eds.), IFIP AICT Vol.326, Springer, 2011, pp.421-427.

[8] Buckl, S., F. Matthes, and C.M. Schweda, "A Viable System Perspective on Enterprise Architecture Management," 2009 IEEE Int Conf SMC, San Antonio, USA, 2009, pp. 1483-1488.

[9] EsmaeilZadeh, M., G. Millar, and E. Lewis, "Mapping the Enterprise Architecture Principles in TOGAF to the Cybernetic Concepts," 45th HICSS IEEE, 2012, pp.42704276.

[10] Beer, S., Decision and Control: The Meaning of Operational Research and Management Cybernetics, New York : Wiley, 1966.

[11] Wiener, N., Cybernetics or Control and Communication in the Animal and the Machine, (2nd Rev. Ed 1961. Cambridge, MA : MIT Press, 1948. 
[12] Ashby, W. R., An introduction to cybernetics, London : Chapman \& Hall, 1956.

[13] Beer, S., Cybernetics and management, Wiley, 1959.

[14] Jackson, M. C., Systems approaches to management, New York : Kluwer /Plenum Publishers, 2000.

[15] Ramage, M. and K. Shipp, Systems thinkers, Springer, 2009.

[16] Schmidt, C. and P. Buxmann, "Outcomes and success factors of enterprise IT architecture management: empirical insight from the international financial services industry,", EJIS 20 (2), 2011, pp. 168-185.

[17] Checkland, P., Systems thinking, Oxford Univ Press, 1999.

[18] Boulding, K. E., "General systems theory-the skeleton of science," Management Science (2:3), 1956, pp. 197-208.

[19] Anderton, R. H. and P. B. Checkland, "On learning our lessons," Internal Discussion Paper, Lancaster, UK: Department of Systems, University of Lancaster, 2/77, 1977.

[20] Checkland, P., Systems Thinking, Systems Practice, Chichester, UK : John Wiley \& Sons, 1996.

[21] Beer, S., The Heart of Enterprise: the Managerial Cybernetics of Organization, New York : Wiley, 1979.

[22] Beer, S., Brain of the Firm, 2nd Ed. Wiley, 1981.

[23] Kandjani, H. and P. Bernus, "Engineering SelfDesigning Enterprises as Complex Systems Using Extended Axiomatic Design Theory," IFAC Papers On Line, IFACWCC18 (1), 2011b, pp. 11943-11948.

[24] Ashby, W. R., "Adaptiveness and equilibrium," The British J of Psychiatry (86:362), 1940, pp. 478-483.

[25] Conant, R. C. and W. R. Ashby, "Every Good Regulator of a System Must be a Model of That System," International Journal of Systems Science (1:2), 1970, pp. 89-97.

[26] Badalotti, E., "New Economy, Complexity and Management. Complexity Theory and the Management of Networks." In Workshop on Organisational Networks as Distributed Systems of Knowledge. P. Andriani and G. Passiante (eds.). Imperial College Press, 2004, pp.59-64.

[27] Henderson J. C. and N. Venkatraman, "Strategic alignment: Leveraging information technology for transforming organizations," IBM Systems Journal, vol. 32, 1993, pp. 4-16.

[28] Suh, N.P., The Principles of Design, New York : Oxford University Press, 1990.

[29] Suh, N.P., Axiomatic design: advances and applications, New York : Oxford University Press, 2001.

[30] Suh, N. P., Complexity: Theory and Applications, New York : Oxford University Press, 2005.

[31] Ashby, W. R., Design for a brain; the origin of adaptive behavior. New York : Wiley, 1960.
[32] Geoghegan, M. C. and P. Pangaro. Design for a selfregenerating organization. International Journal of General Systems (38:2), 2009, pp. 155-173.

[33] Umpleby, S. A., "Ross Ashby's general theory of adaptive systems," Int J of General Systems (38:2), 2009, pp. 231-238.

[34] Bernus, P. and L. Nemes, “A Framework to Define a Generic Enterprise Reference Architecture and Methodology" Computer Integrated Manufacturing Systems (9:3), 1996, pp. 179-191.

[35] ISO15704, "Industrial automation systems Requirements for enterprise-reference architectures and methodologies," Geneva : ISO TC184.SC5.WG1. Geneva, ISO TC184.SC5.WG1, 2000, Amd. 2005. (Annex A, GERAM) also available at www.ict.griffith.edu.au/ bernus/taskforce/geram/versions/

[36] Noran, O., “A Mapping of Individual Architecture Frameworks (GRAI, PERA, C4ISR, CIMOSA, Zachman, ARIS) onto GERAM,” In P. Bernus, et al. (Eds.), Handbook of Enterprise Architecture, Springer Verlag, Heidelberg, 2003, pp. 65-210.

[37] Bernus, P., L. Nemes, and T.J. Williams (eds.), "Architectures for Enterprise Integration," London : Chapman and Hall, 368p, 1996.

[38] Mitleton-Kelly E. Co-Evolutionary Integration: A Complexity Perspective on Mergers \& Acquisitions. [retrieved Aug 2012] from http://www.psych.lse.ac.uk/complexity/ICoSS/Papers/ co_EvolutionaryIntegration.pdf] 2004

[39] Iacocca Institute (1991). 21st Century Manufacturing Enterprise Strategy. An Industry-Led View. Volumes 1 \& 2. Iacocca Institute, Bethlehem, PA.

[40] Goldman, S.L., Nagel, R.N. and Preiss K. (1995). Agile Competitors and Virtual Organizations: Strategies for Enriching the Customer. Van Nostrand Reinhold.

[41] Fine, Ch.H., Freund,R.M. Optimal Investment in Product-flexible manufacturing capacity Management Science . 36 (4) 1990. pp449-466

[42] Stalk, G.Jr. Time - The next source of competitive advantage. Harvard Business Review 66(4) 1988. pp 41-51

[43] A cartridged piping-connecting apparatus, and connecting system using the same. European Patent EP0567986. 1997

[44] Li, M., Vitányi, P. (2008). An introduction to Kolmogorov complexity and its applications. ( $\left.3^{\text {rd }} \mathrm{Ed}\right)$ Springer

[45] Kandjani, H., Bernus,P., Wen, L. Enterprise Architecture Cybernetics for Collaborative Networks: Reducing the Structural Complexity and Transaction Cost via Virtual Brokerage. In Information Control Problems in Manufacturing. IFAC Papers Online. 14(1). 2012. pp12331239 